\title{
Some Generalized Hom-Algebra Structures
}

\section{Bakayoko I* and Diallo OW}

Department of Mathematics, UJNK/Nzerekore University Centre, PO Box 50, Nzerekore, Guinea

\begin{abstract}
In this paper, we introduce generalized left-Hom-symmetric algebras and generalized Hom-dendriform algebras as well as the corresponding modules. We investigate the connection between these categories of generalized Homalgebras and modules. We give various constructions of these generalized Hom-algebra structures from either a given one or an ordinary one. We prove that any generalized Hom-dialgebras give rise to generalized Hom-LeibnizPoisson algebras and generalized Hom-Poisson dialgebras.
\end{abstract}

Keywords: Generalized Hom-associative; Hom-lie; Homdendriform; Hom-Leibniz-Poisson algebras; Generalized Homdialgebras; Graded modules

\section{Introduction}

Hom-algebraic structures were appeared for the first time in the works of Aizawa and Sato [1] as a generalization of Lie algebras. Other interesting Hom-type algebraic structures of many classical structures were studied as Hom-associative algebras, Hom-Lie admissible algebras and more general G-Hom-associative algebras [2], n-ary Hom-Nambu-Lie algebras [3], Hom-Lie admissible Hom-coalgebras and Hom-Hopf algebras [4], Hom-alternative algebras, Hom-Malcev algebras and Hom-Jordan algebras [5], $L$-modules, $L$-comodules and Hom-Lie quasi-bialgebras [6], Laplacian of Hom-Lie quasi- bialgebras [7]. Hom-algebraic structures were extended to the case of G-graded Lie algebras by studying Hom-Lie superalgebras, Hom-Lie admissible superalgebras [8], color Hom-Lie algebras [9], color Hom-Lie bialgebras and color Hom-Poisson bialgebras [10] and color HomPoisson algebras. Color Hom-Poisson algebras were introduced [11] as generalization of Hom-Poisson algebras [2]. It is shown that every color Hom-associative algebra has a non-commutative Hom-Poisson algebra structure in which the Hom-Poisson bracket is the commutator bracket. It is also proved that twisting a color Hom-Poisson module structure map by a color Hom-Poisson algebra endomorphism, we get another one. Color Hom-Poisson algebras generalize color Homassociative algebras $[9,12]$ color Hom-Lie algebras $[9,12]$ and HomLie superalgebras [13]. The author [12] presents some constructions of quadratique color Hom-Lie algebras, and this is used to provide several examples. $T^{*}$-extensions and central extensions of color Hom-Lie algebras and some cohomological characterizations are established [14]. These structures are well-known to physicists and to mathematicians studying differential geometry and homotopy theory. The cohomology theory of Lie superalgebras [15] has been generalized to the cohomology of Hom-Lie superalgebras [16].

The purpose of this paper is to introduce some generalized (color or graded) Hom-algebras and the corresponding modules. The paper is organized as follows. In section 2, we recall some basic notions related to color Hom-associative algebras and color HomLie algebras. In section 3 , we define generalized left-Hom-symmetric algebras (resp. generalized Hom-dendriform algebras) and prove that to any generalized left-Hom-symmetric algebra one may associate a generalized Hom-Lie algebra (resp. a generalized Hom-dendriform algebra and a generalized Hom-associative algebra). Next, we prove that any generalized left-symmetric algebra can be deformed into a generalized left-Hom-symmetric algebra along with any even linear selfmap. In section 4, we point out that generalized Hom-dialgebras give rise to generalized non-commutative Hom-Leibniz-Poisson algebras and generalized Hom-Poisson dialgebras. A construction theorem of generalized Hom-Poisson dialgebras from generalized Poisson dialgebras and an endomorphism is given. In section 5 , we point out that bimodules over a generalized left-Hom-symmetric algebra $S$ (resp. generalized Hom-dendriform algebra $D$ ) extend to modules over the generalized Hom-Lie algebra (resp. bimodules over the generalized left-Hom-symmetric algebra, generalized Hom-associative algebra) associated to $S$ (resp. $D$ ).

Throughout this paper, all graded vector spaces are assumed to be over a field $\mathbf{K}$ of characteristic different from

\section{Preliminaries}

Let $G$ be an abelian group. A vector space $V$ is said to be a G-graded if, there exists a family $(V) \in_{G}$ of vector subspaces of $V$ such that

$$
V=\bigoplus_{a \in G} V_{a} .
$$

An element $x \in V$ is said to be homogeneous of degree $a \in G$ if $x \in$ $V_{a}$. We denote $\mathrm{H}(\mathrm{V})$ the set of all homogeneous elements in $V$.

Let $V=\oplus_{a \in G} V_{a}$ and $V^{\prime}=\oplus_{a \in G} V_{a}^{\prime}$ be two $G$-graded vector spaces. A linear mapping $f: V \rightarrow V^{\prime}$ is said to be homogeneous of degree $b$ if

$$
f\left(V_{a}\right) \subseteq V_{a+b}^{\prime}, \forall a \in G
$$

If, $f$ is homogeneous of degree zero i.e. $f\left(V_{a}\right) \subseteq V_{a}^{\prime}$ holds for any a $\in \mathrm{G}$, then $f$ is said to be even.

An algebra $(A, \mu)$ is said to be $G$-graded if its underlying vector space is $G$-graded i.e. $A=\bigoplus_{a \in G} A_{a}$, and if furthermore $\mu\left(A_{a}, A_{b}\right) \subseteq A_{a+b}$, for all $a, b \in G$. Let be another $G$-graded algebra. A morphism $f: A \rightarrow A^{\prime}$ of $G$-graded algebras is by definition an algebra morphism from $A$ to $A^{\prime}$ which is, in addition an even mapping.

Definition 2.1: Let $G$ be an abelian group. A map $\varepsilon: G \times G \rightarrow \mathbf{K}^{*}$ is

${ }^{*}$ Corresponding author: Bakayoko I, Department of Mathematics, UJNK Nzerekore University Centre, PO Box 50, Nzerekore, Guinea, Tel: 224631945919 ; E-mail: ibrahimabakayoko27@gmail.com

Received January 23, 2015; Accepted July 25, 2015; Published July 29, 2015

Citation: Bakayoko I, Diallo OW (2015) Some Generalized Hom-Algebra Structures. J Generalized Lie Theory Appl 9: 226. doi:10.4172/1736-4337.1000226

Copyright: $\odot 2015$ Bakayoko I, et al. This is an open-access article distributed under the terms of the Creative Commons Attribution License, which permits unrestricted use, distribution, and reproduction in any medium, provided the original author and source are credited. 
called a skew-symmetric bicharacter on $G$ if the following identities hold,

(i) $\varepsilon(a, b) \varepsilon(b, a)=1$,

(ii) $\varepsilon(a, b+c)=\varepsilon(a, b) \varepsilon(a, c)$,

(iii) $\varepsilon(a+b, c)=\varepsilon(a, c) \varepsilon(b, c)$,

$\mathrm{a}, \mathrm{b}, \mathrm{c} \in \mathrm{G}$,

Remark that

$\varepsilon(a, 0)=\varepsilon(0, a)=1, \varepsilon(a, a)= \pm 1 \quad$ for all $a \in G$,

where 0 is the identity of $G$.

If $x$ and $y$ are two homogeneous elements of degree $a$ and $b$ respectively and $\varepsilon$ is a skew- symmetric bicharacter, then we shorten the notation by writing $\varepsilon(x, y)$ instead of $\varepsilon(a, b)$.

Definition 2.2: A generalized Hom-associative algebra is a quadruple $(A, \mu, \varepsilon, \alpha)$ consisting of a G-graded vector space $A$, an even bilinear map $\mu: A \times A \rightarrow A$, a bicharacter $\varepsilon$ and an even linear map $\alpha: A$ $\rightarrow$ A such that

$$
\mu(\alpha(x), \mu(y, z))=\mu(\mu(x, y), \alpha(z)) .
$$

If in addition $\mu(x, y)=\varepsilon(x, y) \mu(y, x)$, the generalized Hom-associative algebra $(A, \mu, \varepsilon, \alpha)$ is said to be a $\varepsilon$-commutative generalized Homassociative algebra.

Remark 2.1: When $\alpha=I d$, we recover the classical generalized associative algebra.

Recall that the (generalized) Hom-associator, $a s_{A}$, of a Hom-algebra $A$ is defined as

$a s_{A}: A \otimes A \otimes A \rightarrow A, \quad(x, y, z) \mapsto \mu(\mu(x, y), \alpha(z))-\mu(\alpha(x), \mu(y, z))$.

Observe that $a s_{A} \equiv 0$ when $A$ is a generalized Hom-associative algebra.

Definition 2.3: A generalized Hom-Lie algebra is a quadruple (A, $[-,-], \varepsilon, \alpha)$ consisting of a $G$-graded vector space $A$, an even bilinear map $[-,-]: A \times A \rightarrow A$ (i.e. $\left[A_{a^{\prime}} A_{b}\right] \subseteq A_{a+b}$ for all $\left.a, b \in G\right)$, a bicharacter $\mathcal{E}$, and an even linear map $\alpha: A \rightarrow A$ such that for any $x, y \in \mathcal{H}(A)$ [12], we have

$$
[x, y]=-\varepsilon(x, y)[y, x] \quad(\varepsilon \text {-skew-symmetry }),
$$

$\alpha \mathrm{o}[-,-]=[-,-] \mathrm{o} \alpha^{\otimes 2} \quad$ (multiplicativity),

$\oint \varepsilon(z, x)[\alpha(x),[y, z]]=0 \quad(\varepsilon$-Hom-Jacobi identity $)$,

Where $\oint$ means cyclic summation.

Remark 2.2: A generalized Lie algebra $(A,[-,-], \varepsilon)$ is a generalized Hom-Lie algebra with $\alpha=I d$.

Examples of generalized Hom-Lie algebras are provided $[9,12]$.

\section{Generalized Left-Hom-symmetric Algebras and Generalized Hom-dendriform Algebras}

This section is devoted to generalized left-Hom-symmetric algebras and generalized Hom- dendriform algebras which are the twisted analogue of generalized left-symmetric algebras [17] and Homdendriform algebras $[18,19]$ respectively.

The following lemma connects generalized Hom-associative algebras to generalized Hom-Lie algebras.
Lemma 3.1: Let $(A, \mu, \varepsilon, \alpha)$ be a generalized Hom-associative algebra [12].

Then $\left(A,[\cdot, \cdot]=\mu-\varepsilon(\cdot, \cdot) \mu^{o p}, \varepsilon, \alpha\right)$ is a generalized Hom-Lie algebra, denoted by $L(A)$.

Definition 3.1: Let $G$ be an abelian group and $\varepsilon$ a bicharacter on $G$. A graded non-associative algebra $S=\oplus_{a \in G} S_{a}$ with the even linear map $\alpha: S \rightarrow S$ and the multiplication $(x, y) \mapsto x^{\circ} y$ satisfying

$$
S_{a}^{\circ} S_{b} \subseteq S_{a+b}
$$

is called a generalized left-Hom-symmetric algebra if the following generalized left-Hom- symmetric identity (or E-left-Hom-symmetric identity) is satisfied

$(x \circ y) \circ \alpha(z)-\alpha(x) \circ(y \circ z)=\varepsilon(x, y)((y \circ x) \circ \alpha(z)-\alpha(y) \circ(x \circ z))$,

for all $x, y, z \in \mathcal{H}(S)$.

In term of the (generalized) Hom-associator, the $\varepsilon$-left-Homsymmetric identity may written as

$$
\operatorname{alph} a(x, y, z)=\varepsilon(x, y) a s_{\alpha}(y, x, z)
$$

Definition 3.2: Let $(S, \mu, \varepsilon, \alpha)$ and $\left(S^{\prime}, \mu^{\prime}, \varepsilon^{\prime}, \alpha^{\prime}\right)$ be two generalized Hom-left-symmetric algebras. An even linear map $f: S \rightarrow S^{\prime}$ is said to be a morphism of generalized Hom-left-symmetric algebras if $f$ o $\alpha=\alpha^{\prime}$ o $f$ and

$f(\mu(x, y))=\mu^{\prime}(f(x), f(y))$,

for all $x, y \in S$.

The following result allows to get a generalized left-Hom-symmetric algebra from a given one.

Theorem 3.1: Let $(S, \bullet, \varepsilon, \alpha)$ be a generalized left-Hom-symmetric algebra and $\beta: A \rightarrow A$ a left-Hom-symmetric algebra endomorphism. Then, for any non-negative entiger $n, A_{\beta}=\left(A,{ }_{\beta}{ }_{\beta}=\beta^{n} \mathrm{o} \cdot \varepsilon, \beta^{n} \mathrm{o} \alpha\right)$ is a generalized left-Hom-symmetric algebra, where $\beta^{n}=\beta^{\circ} \beta^{n-1}$.

Moreover, suppose that $\left(A^{\prime}, \bullet^{\prime}, \varepsilon\right)$ is another generalized leftsymmetric algebra and $\alpha^{\prime}: A^{\prime} \rightarrow A^{\prime}$ a left-Hom-symmetric algebras endorphism. If $\alpha^{\prime}: A^{\prime} \rightarrow A^{\prime}$ is a generalized left-symmetric algebras morphism that satisfies $f$ o $\beta^{n}=\alpha^{\prime n}$ o $f$, then

$$
f:\left(A, \bullet^{\prime}=\beta^{n} o^{\bullet}, \varepsilon, \beta^{n} o \alpha\right) \rightarrow\left(A^{\prime}, \bullet^{\prime} \alpha^{\prime n}=\alpha^{\prime n} o^{\bullet^{\prime}}, \varepsilon, \alpha^{\prime n}\right)
$$

is a morphism of generalized left-Hom-symmetric algebras.

Proof: For any $x, y, z \in \mathcal{H}(S)$,

$$
\begin{aligned}
& \operatorname{as}_{\beta}(x, y, z):=(x \bullet \beta y) \bullet_{\beta}\left(\beta^{n^{\circ}} \alpha\right)(z)-\left(\beta^{n \circ} \alpha\right)(x) \bullet_{\beta}\left(y \bullet_{\beta} z\right) \\
& =\beta^{n}\left(\beta^{n}(x \cdot y) \bullet\left(\beta^{n \circ} \alpha\right)(z)\right)-\beta^{n}\left(\left(\beta^{n \circ} \alpha\right)(x) \cdot \beta^{n}(y \cdot z)\right) \\
& =\beta^{2 n}((x \cdot y) \cdot \alpha(z)-\alpha(x) \cdot(y \cdot z)) \\
& =\varepsilon(x, y) \beta^{2 n}((y \cdot x) \cdot \alpha(z)-\alpha(y) \cdot(x \cdot z)) \\
& =\varepsilon(x, y) \beta^{n}\left(\beta^{n}(y \cdot x) \cdot\left(\beta^{n \circ} \alpha\right)(z)-\left(\beta^{n \circ} \alpha\right)(y) \cdot \beta^{n}(x \cdot z)\right) \\
& =\varepsilon(x, y)\left(\left(y \bullet_{\beta} x\right) \bullet_{\beta}\left(\beta^{n \circ} \alpha\right)(z)-\left(\beta^{n \circ} \alpha\right)(y) \bullet_{\beta}\left(x \bullet_{\beta} z\right)\right) \\
& =\varepsilon(x, y) a s_{\beta}(y, x, z) .
\end{aligned}
$$

For the second part, we have

$f(x \bullet \beta)=f \beta^{n}(x \bullet y)=\alpha^{\prime n} f(x \bullet y)=\alpha^{\prime n}\left(f(x) \bullet^{\prime} f(y)\right)=f(x){ }^{\prime}{ }^{\prime n} f(y)$ 
This completes the proof.

Lemma 3.2: Let $(S, \circ, \varepsilon, \alpha)$ be a generalized left-Hom-symmetric algebra. Then, for any $x, y \in \mathcal{H}(S)$, the commutator

$[x, y]=x^{\circ} y-\varepsilon(x, y) y^{\circ} x$

makes $S$ to become a generalized Hom-Lie algebra, denoted $L(S)$.

Proof: For any $x, y, z \in \mathcal{H}(S)$, we have

$\oint \varepsilon(z, x)[\alpha(x),[y, z]]=\underbrace{\varepsilon(z, x) \alpha(x)^{\circ}\left(y^{\circ} z\right)}_{a_{1}}-\underbrace{\varepsilon(z, x) \varepsilon(y, z) \alpha(x)^{\circ}\left(z^{\circ} y\right)}_{b_{1}}-\underbrace{\varepsilon(x, y)\left(y^{\circ} z\right)^{\circ} \alpha(x)}_{c_{1}}$ $+\underbrace{\varepsilon(x, y) \varepsilon(y, z)\left(z^{\circ} y\right)^{\circ} \alpha(x)}_{c_{2}}+\underbrace{\varepsilon(x, y) \alpha(y)^{\circ}\left(z^{\circ} x\right)}_{c_{3}}-\underbrace{\varepsilon(x, y) \varepsilon(z, x) \alpha(y)^{\circ}\left(x^{\circ} z\right)}_{a_{2}}$ $-\underbrace{\varepsilon(y, z)\left(z^{\circ} x\right)^{\circ} \alpha(y)}_{b_{2}}+\underbrace{\varepsilon(y, z) \varepsilon(z, x)\left(x^{\circ} z\right)^{\circ} \alpha(y)}_{b_{3}}+\underbrace{\varepsilon(y, z) \alpha(z)^{\circ}\left(x^{\circ} y\right)}_{b_{4}}$

$-\underbrace{\varepsilon(y, z) \varepsilon(x, y) \alpha(z)^{\circ}\left(y^{\circ} x\right)}_{c_{4}}-\underbrace{\varepsilon(z, x)\left(x^{\circ} y\right)^{\circ} \alpha(z)}_{a_{3}}+\underbrace{\varepsilon(z, x) \varepsilon(x, y)\left(y^{\circ} x\right)^{\circ} \alpha(z)}_{a_{4}}$

Using the $\varepsilon$-left-Hom-symmetric identity, observe that the following sums are $0: a_{1}+a_{2}+a_{3}+a_{4}, b_{1}+b_{2}+b_{3}+b_{4}$ and $c_{1}+c_{2}+c_{3}+$ $c_{4}$. This shows that $\oint \varepsilon(z, x)[\alpha(x),[y, z]]=0$.

Definition 3.3: A generalized Hom-dendriform algebra is a $G$-graded vector space $D$ together with two even bilinear maps $\dashv: D \otimes D \rightarrow D, \vdash: D \otimes D \rightarrow D$ and an even linear map $\alpha: S \rightarrow S$ such that

$$
\begin{aligned}
& \alpha(x) \vdash(y \dashv z)=\varepsilon(x, z)(x \vdash y) \dashv \alpha(z), \\
& (x \dashv y) \dashv \alpha(z)=\varepsilon(z, y) \alpha(x) \dashv(y \vdash z)+\alpha(x) \dashv(y \dashv z), \\
& \alpha(x) \vdash(y \vdash z)=\varepsilon(x, y)(x \dashv y) \vdash \alpha(z)+(x \vdash y) \vdash \alpha(z) .
\end{aligned}
$$

If $\left(D^{\prime}, \dashv^{\prime}, \vdash^{\prime}, \varepsilon, \alpha^{\prime}\right)$ is another generalized Hom-dendriform algebra and $f: D \rightarrow D^{\prime}$ is an even linear map such that $f^{\circ} \alpha=\alpha^{\circ \circ} f$, then $f$ is said to be a morphism of generalized Hom-dendriform algebras when it is compatible with both products $\dashv$ and $\vdash$

When $\alpha=I d$, we obtain morphism of generalized dendriform algebras.

In a generalized Hom-dendriform algebra $(D, \dashv, \vdash, \varepsilon, \alpha)$, if the twisting map $\alpha$ is morphism, then $(D, \dashv, \vdash, \varepsilon, \alpha)$ is said to be a multiplicative generalized Hom-dendriform algebra.

The below Theorem produces generalized Hom-dendriform algebras from generalized dendriform algebras.

Theorem 3.2: Let $(D, \dashv, \vdash, \varepsilon)$ be a generalized dendriform algebra and $\alpha: D \rightarrow D$ an even linear map such that

$\dashv{ }^{\circ}(\alpha \otimes \alpha)=\alpha^{\circ} \dashv$ and $\vdash^{\circ}(\alpha \otimes \alpha)=\alpha^{q}$.

Then, for any non-negative entiger $n,\left(D, \dashv_{n}=\alpha^{n} \circ-, \vdash_{n}=\alpha^{n} \bigcirc-, \varepsilon, \alpha^{n}\right)$ is a generalized multiplicative Hom-dendriform algebra.

Suppose that $\left(D^{\prime}, \dashv^{\prime}, \vdash^{\prime}, \varepsilon^{\prime}\right)$ is another generalized dendriform algebra and $\alpha^{\prime}: D^{\prime} \rightarrow D^{\prime}$ an even linear map that satisfies $\dashv^{\prime \circ}\left(\alpha^{\prime} \otimes \alpha^{\prime}\right)=\alpha^{\prime \circ} \dashv^{\prime}$ and $\vdash^{\prime}{ }^{\circ}\left(\alpha^{\prime} \otimes \alpha^{\prime}\right)=\alpha^{\prime \circ} \vdash^{\prime}$. If $f: D \rightarrow D^{\prime}$ is a morphism of generalized dendriform algebras, then $f:\left(D, \dashv_{n}, \vdash_{n}, \varepsilon, \alpha^{n}\right) \rightarrow\left(D^{\prime},-_{n}^{\prime}, \vdash_{n}^{\prime}, \varepsilon, \alpha^{\prime n}\right)$ is a morphism of generalized Hom-dendriform algebra.

Proof: The three axioms are easy to check. For example, for any $x, y, z \in \mathcal{H}(D)$,

$$
\begin{aligned}
& \alpha^{n}(x) \vdash_{n}\left(y \dashv_{n} z\right)=\alpha^{n}\left(\alpha^{n}(x) \vdash \alpha^{n}(y \dashv z)\right)=\alpha^{2 n}(x \vdash(y \dashv z)) \\
& =\varepsilon(x, z) \alpha^{2 n}((x \vdash y) \dashv z)=\varepsilon(x, z) \alpha^{n}\left(\alpha^{n}(x \vdash y) \dashv \alpha^{n} z\right) \\
& =\varepsilon(x, z)\left(x \vdash_{n} y\right) \dashv_{n} \alpha^{n} z .
\end{aligned}
$$

This proves (9). The other two axioms are proved similarly. The second part is showed likewise the proof of the second part of Theorem 3.1.

Lemma 3.3: Let $(D, \dashv, \vdash, \varepsilon, \alpha)$ be a generalized Hom-dendriform algebra. Then, $D$ is a generalized left-Hom-symmetric algebra with the structure map

$$
\begin{aligned}
& x^{\circ} y=x \dashv y+y \vdash x \\
& x, y \in D .
\end{aligned}
$$

Proof: For any $x, y, z \in D$, we have

$\left(x^{\circ} y\right)^{\circ} \alpha(z)-\alpha(x)^{\circ}\left(y^{\circ} z\right)-\varepsilon(x, y)\left(\left(y^{\circ} x\right)^{\circ} \alpha(z)-\alpha(y)^{\circ}\left(x^{\circ} z\right)\right)=$

$=(x \vdash y-y \dashv x)^{\circ} \alpha(z)-\alpha(x)^{\circ}(y \vdash z-z \dashv y)$

$-\varepsilon(x, y)(y \vdash x-x \dashv y)^{\circ} \alpha(z)+\varepsilon(x, y) \alpha(y)^{\circ}(x \vdash z-z \dashv x)$

$=(x \vdash y-y \dashv x) \vdash \alpha(z)-\alpha(z) \dashv(x \vdash y-y \dashv x)-\alpha(x) \vdash(y \vdash z-z \dashv y)$

$+(y \vdash z-z \dashv y) \dashv \alpha(x)-\varepsilon(x, y)(y \vdash x-x \dashv y) \vdash \alpha(z)+\varepsilon(x, y) \alpha(z) \dashv(y \vdash x-x \dashv y)$

$+\varepsilon(x, y) \alpha(y) \vdash(x \vdash z-z \dashv x)-\varepsilon(x, y)(x \vdash z-z \dashv x) \dashv \alpha(y)$

$=\underbrace{(x \vdash y) \vdash \alpha(z)}_{a_{1}}-\underbrace{(y \dashv x) \vdash \alpha(z)}_{b_{1}}-\underbrace{\alpha(z) \dashv(x \vdash y)}_{c_{1}}+\underbrace{\alpha(z) \dashv(y \dashv x)}_{d_{1}}-\underbrace{\alpha(x) \vdash(y \vdash z)}_{a_{2}}$

$+\underbrace{\alpha(x) \vdash(z \dashv y)}_{e_{1}}+\underbrace{(y \vdash z) \dashv \alpha(x)}_{f_{1}}-\underbrace{(z \dashv y) \dashv \alpha(x)}_{d_{2}}-\underbrace{\varepsilon(x, y)(y \vdash x) \vdash \alpha(z)}_{b_{2}}$

$+\underbrace{\varepsilon(x, y)(x \dashv y) \vdash \alpha(z)}_{a_{3}}+\underbrace{\varepsilon(x, y) \alpha(z) \dashv(y \vdash x)}_{d_{3}}-\underbrace{\varepsilon(x, y) \alpha(z) \dashv(x \dashv y)}_{c_{2}}+\underbrace{\varepsilon(x, y) \alpha(y) \vdash(x \vdash z)}_{b_{3}}$

$-\underbrace{\varepsilon(x, y) \alpha(y) \vdash(z \dashv x)}_{f_{2}}-\underbrace{\varepsilon(x, y)(x \vdash z) \dashv \alpha(y)}_{e_{2}}+\underbrace{\varepsilon(x, y)(z \dashv x) \dashv \alpha(y)}_{c_{3}}$.

The term $a_{1}+a_{2}+a_{3} \operatorname{vanish}(11), b_{1}+b_{2}+b_{3}$ vanish (11), $c_{1}+c_{2}$ $+c_{3}$ vanishes (10), $d_{1}+d_{2}+d_{3}$ vanishes $(10), e_{1}+e_{2}$ vanishes $(9), f_{1}$ $+f_{2}$ vanishes (9). Thus, $\left(D,{ }^{\circ}, \varepsilon, \alpha\right)$ is a generalized Hom-left-symmetric algebra.

Lemma 3.4: Let $(D, \dashv, \vdash, \varepsilon, \alpha)$ be a generalized Hom-dendriform algebra. Then, $(D, \star, \alpha)$ is a generalized Hom-associative algebra with the multiplication given by

$x \star y=y \vdash x+(x, y) x \dashv y$

$x, y \in \mathcal{H}(D)$

Proof: For any $x, y, z \in \mathcal{H}(D)$,

$$
\begin{aligned}
& a s_{\alpha}(x, y, z)=(x \star y) \star \alpha(z)-\alpha(x) \star(y \star z) \\
= & (x \vdash y+\varepsilon(x, y) x \dashv y) \star \alpha(z)-\alpha(x) \star(y \vdash z+\varepsilon(y, z) y \dashv z) \\
= & (x \vdash y+\varepsilon(x, y) x \dashv y) \vdash \alpha(z)+\varepsilon(x+y, z)(x \vdash y+\varepsilon(x, y) x \dashv y) \dashv \alpha(z) \\
- & \alpha(x) \vdash(y \vdash z+\varepsilon(y, z) y \dashv z)-\varepsilon(x, y+z) \alpha(x) \dashv(y \vdash z+\varepsilon(y, z) y \dashv z) \\
= & (x \vdash y) \vdash \alpha(z)+\varepsilon(x, y)(x \dashv y) \vdash \alpha(z)+\varepsilon(x, z) \varepsilon(y, z)(x \vdash y) \dashv \alpha(z) \\
+ & \varepsilon(x, z) \varepsilon(y, z) \varepsilon(x, y)(x \dashv y) \dashv \alpha(z)-\alpha(x) \vdash(y \vdash z)-\varepsilon(y, z) \alpha(x) \vdash(y \dashv z) \\
- & \varepsilon(x, y) \varepsilon(x, z) \alpha(x) \dashv(y \vdash z)-\varepsilon(x, y) \varepsilon(x, z) \varepsilon(y, z) \alpha(x) \dashv(y \dashv z) .
\end{aligned}
$$

The left hand side vanishes by axioms in Definition 3.3. Therefore, 
the conclusion holds.

Let us denote by GHDA: the category of generalized Homdendriform algebras, GLHSA: the category of generalized left-Homsymmetric algebras, GHAA: the category of generalized Homassociative algebras and

GHLA: the category of generalized Hom-Lie algebras. Then, the above discussion may be summarized in the following theorem.

Theorem 3.3: The following diagram is commutative

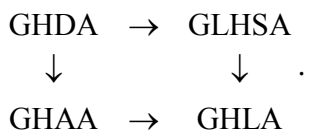

Proof: The top horizontal arrow and the bottom horizontal arrow follow from Lemma 3.3 and Lemma 3.1 respectively. The left vertical arrow is established in Lemma 3.4 and right vertical arrow is the functor constructed in Lemma 3.2.

\section{Generalized Hom-Leibniz-Poisson Algebras and Generalized Hom-Poisson Dialgebras}

In this section we will show that generalized Hom-dialgebras give rise to generalized non- commutative Hom-Leibniz-Poisson algebras and generalized Hom-Poisson dialgebras.

Definition 4.1: A generalized Hom-dialgebra is a quintuple $(D, \dashv, \vdash, \varepsilon, \alpha)$, where $D$ is a G-graded vector space, $\dashv, \vdash: D \otimes D \rightarrow D$ are even bilinear maps, $\varepsilon: G \otimes G \rightarrow K^{*}$ is a bicharacter and $\alpha: D \rightarrow D$ is an even linear map such that the following five axioms are satisfied for $x, y, z \in \mathcal{H}(D)$ :

$$
\begin{aligned}
& (x \vdash y) \dashv \alpha(z) \stackrel{(a)}{=} \alpha(x) \vdash(y \dashv z) \\
& \alpha(x) \dashv(y \dashv z) \stackrel{(b)}{=}(x \dashv y) \dashv \alpha(z) \stackrel{(c)}{=} \alpha(x) \dashv(y \vdash z) \\
& (x \vdash y) \vdash \alpha(z) \stackrel{(d)}{=} \alpha(x) \vdash(y \vdash z) \stackrel{(e)}{=}(x \dashv y) \vdash \alpha(z)
\end{aligned}
$$

Definition 4.2: A generalized non-commutative Hom-LeibnizPoisson algebra is a G-graded vector space $P$ together with two even bilinear maps $[-,-]: P \otimes P \rightarrow P$ and $: P P \otimes P \rightarrow P$, a bicharacter $\varepsilon: G \otimes G \rightarrow K^{*}$ and an an even linear map $\alpha: P \rightarrow P$ such that

i) $(P,[-,-], \varepsilon, \alpha)$ is a generalized Hom-Leibniz algebra i.e.

$$
[[x, y], \alpha(z)]=[\alpha(x),[y, z]]+\varepsilon(y, z)[[x, z], \alpha(y)],
$$

ii) $(P, \cdot, \varepsilon, \alpha)$ is a generalized Hom-associative algebra, iii) and the following identity

$$
[x \cdot y, \alpha(z)]=\alpha(x) \cdot[y, z]+\varepsilon(y, z)[x, z] \cdot \alpha(y),
$$

holds, for all $x, y, z \in \mathcal{H}(P)$.

If in addition, $\alpha$ is an endomorphism with respect to $\cdot$ and [-, $-]$, we say that $(P, ;[-,-], \varepsilon, \alpha)$ is a multiplicative generalized noncommutative Hom-Leibniz-Poisson algebra.

Remark 4.1: When the Hom-associative product $\bullet$ is $\mathcal{E}$-commutative, then $(A, \cdot,[-,-], \varepsilon, \alpha)$ is said to be a commutative generalized HomLeibniz Poisson algebra.

The following result gives a connection generalized Hom-dialgebras and generalized Hom-Leibniz-Poisson algebras.
Theorem 4.1: Let $(D, \dashv, \vdash, \varepsilon, \alpha)$ be a generalized Hom-dialgebra. Define the even bilinear map $[-,-]: D \otimes D \rightarrow D$ by setting

$[x, y]:=x \dashv y-\varepsilon(x, y) y \vdash x$.

Then $(D,-,[-,-], \varepsilon, \alpha)$ is a generalized Hom-Leibniz-Poisson algebra.

Proof: We write down all the twelve terms involved in the HomLeibniz identity :

$[[x, y], \alpha(z)]=(x \dashv y-\varepsilon(x, y) y \vdash x) \dashv \alpha(z)-\varepsilon(x, y+z) \alpha(z) \vdash(x \dashv y-\varepsilon(x, y) y \vdash x)$
$[[x, z], \alpha(y)]=(x \dashv z-\varepsilon(x, z) z \vdash x) \dashv \alpha(y)-\varepsilon(x+z, y) \alpha(y) \vdash(x \dashv z-\varepsilon(x, z) z \vdash x)$
$[\alpha(x),[y, z]]=\alpha(x) \dashv(y \dashv z-\varepsilon(y, z) z \vdash y)-\varepsilon(x, y+z)(y \dashv z-\varepsilon(y, z) z \vdash y) \vdash \alpha(x)$

Using the five axioms in Definition 4.1, it is immediate to see that (12) hols. And,

$$
\begin{aligned}
& {[x \dashv y, \alpha(z)]-\alpha(x) \dashv[y, z]-\varepsilon(y, z)[x, z] \dashv \alpha(y)=} \\
& =(x \dashv y) \dashv \alpha(z)-\varepsilon(x+y, z) \alpha(z) \vdash(x \dashv y)-\alpha(x) \dashv(y \dashv z) \\
& +\varepsilon(y, z) \alpha(x) \dashv(z \vdash y)-\varepsilon(y, z)(x \dashv z-\varepsilon(x, z) z \vdash x) \dashv \alpha(y)
\end{aligned}
$$

The left hand side vanishes by axioms in Definition 4.1.

In the sequel, we define generalized Hom-Poisson dialgebras, and we give a construction theorem.

Definition 4.3: A generalized Hom-Poisson dialgebra is a sextuple $(P, \dashv, \vdash,[-,-], \varepsilon, \alpha)$ in which $P$ is a G-graded vector space $\dashv, \vdash,[-,-]: P \otimes P \rightarrow P$ are three even bilinear maps, $\varepsilon: G \otimes G \rightarrow K^{*}$ is a bicharacter and $\alpha: P \rightarrow P$ is an even linear map such that

$$
\begin{aligned}
& {[x \dashv y, \alpha(z)]=\alpha(x) \dashv[y, z]+\varepsilon(y, z)[x, z] \dashv \alpha(y)} \\
& {[x \vdash y, \alpha(z)]=\alpha(x) \vdash[y, z]+\varepsilon(y, z)[x, z] \vdash \alpha(y)} \\
& {[\alpha(x), y \dashv z]=\varepsilon(x, y) \alpha(y) \vdash[x, z]+[x, y] \dashv \alpha(z)=[\alpha(x), y \vdash z] .} \\
& \text { for all } x, y, z \in \mathcal{H}(P) .
\end{aligned}
$$

Example 4.1: Any Hom-Poisson dialgebra [20] is a generalized Hom-Poisson dialgebra with trivial grading.

Theorem 4.2: Let $(D, \dashv, \vdash, \varepsilon, \alpha)$ be a generalized Hom-dialgebra. Then $(D,-\dashv, \vdash,[-,-], \varepsilon, \alpha)$ is a generalized Hom-Poisson dialgebra, where

$$
[x, y]=x \dashv y-\varepsilon(x, y) y \vdash x,
$$

for any $x, y \in \mathcal{H}(D)$.

Proof: According to the second part of the proof of Theorem 4.1, we only need to prove (15) and (16). For any $x, y, z \in \mathcal{H}(D)$, we have

$$
\begin{aligned}
\alpha(x) \vdash[y, z]+ & \varepsilon(y, z)[x, z] \vdash \alpha(y)=\alpha(x) \vdash(y \dashv z)-\varepsilon(y, z) \alpha(x) \vdash(z \vdash y) \\
& +\varepsilon(y, z)(x \dashv z) \vdash \alpha(y)-\varepsilon(y, z) \varepsilon(x, z)(z \vdash x) \vdash \alpha(y) \\
& =\alpha(x) \vdash(y \dashv z)-\varepsilon(x+y, z)(z \vdash x) \vdash \alpha(y) \quad(\text { by }(e)) \\
& =(x \vdash y) \dashv \alpha(z)-\varepsilon(x+y, z) \alpha(z) \vdash(x \vdash y) \quad(\text { by }(a) \text { and }(d)) \\
& =[x \vdash y, \alpha(z)] .
\end{aligned}
$$

$\varepsilon(x, y) \alpha(y) \vdash[x, z]+[x, y] \dashv \alpha(z)=\varepsilon(x, y) \alpha(y) \vdash(x \dashv z)-\varepsilon(x, y) \varepsilon(x, z) \alpha(y) \vdash(z \vdash x)$

$$
\begin{aligned}
& +(x \dashv y) \dashv \alpha(z)-\varepsilon(x, y)(y \vdash x) \dashv \alpha(z) \\
& =(x \dashv y) \dashv \alpha(z)-\varepsilon(x, y+z) \alpha(y) \vdash(z \vdash x) \quad \text { by }(a)) \\
& =\alpha(x) \dashv(y \dashv z)-\varepsilon(x, y+z)(y \dashv z) \vdash \alpha(x) \quad(\text { by }(b) \text { and }(e)) \\
& =\alpha(x), y \dashv z] .
\end{aligned}
$$


Citation: Bakayoko I, Diallo OW (2015) Some Generalized Hom-Algebra Structures. J Generalized Lie Theory Appl 9: 226. doi:10.4172/17364337.1000226

Page 5 of 7

$\varepsilon(x, y) \alpha(y) \vdash[x, z]+[x, y] \dashv \alpha(z)=(x \dashv y) \dashv \alpha(z)-\varepsilon(x, y+z) \alpha(y) \vdash(z \vdash x)$ $=\alpha(x) \dashv(y \vdash z)-\varepsilon(x, y+z)(y \vdash z) \vdash \alpha(x)$ (by $(c)$ and $(d))$ $=[\alpha(x), y \vdash z]$.

This completes the proof.

Definition 4.4: Let $(P, \dashv, \vdash,[-,-], \varepsilon, \alpha)$ and $\left(D^{\prime}, \dashv^{\prime}, \vdash^{\prime},[-,-]^{\prime}, \varepsilon, \alpha^{\prime}\right)$ be two Hom-Poisson dialgebras. An even linear map $f: D \rightarrow D^{\prime}$ is said to a morphism of generalized Hom-Poisson dialgebras, if $\alpha^{\circ \circ} f=f^{\circ} \alpha$ and for any $x, y \in \mathcal{H}(P)$,

$$
f(x \dashv y)=f(x) \dashv^{\prime} f(y), f(x \vdash y)=f(x) \vdash^{\prime} f(y), f([x, y])=[f(x), f(y)]^{\prime}
$$

The following theorem allows obtaining a generalized Hom-Poisson dialgebra from generalized Poisson dialgebra and an endomorphism.

Proposition 4.1: Let $(D, \dashv, \vdash,[-,-], \varepsilon)$ be a generalized Poisson dialgebra and $\alpha: D \rightarrow D$ an endomorphism of generalized Poisson dialgebras. Then, $\left(D, \dashv_{\alpha}, \vdash_{\alpha},[-,-]_{\alpha}, \varepsilon, \alpha\right)$ is a generalized Hom-Poisson dialgebra, with

$x \dashv_{\alpha} y=\alpha(x \dashv y), x \vdash_{\alpha} y=\alpha(x \vdash y),[x, y]_{\alpha}=\alpha([x, y])$,

for all $x, y \in \mathcal{H}(P)$.

Proof: The proof is analogue to the one of Theorem 3.2.

\section{Bimodules Over Generalized Hom-dendriform and Generalized Left-Hom-symmetric Algebras}

In this section, we introduce modules over some generalized Homalgebras and study the connection between them.

Recall the following definitions.

Definition 5.1: Let $G$ be an abelian group. A Hom-module is a pair $(M, \beta)$ in which $M$ is a G-graded vector space and $\beta: M \rightarrow M$ is an even linear map [11].

Definition 5.2: Let $(A, \mu, \varepsilon, \alpha)$ be a generalized Hom-associative algebra. An A-module is a Hom-module $(M, \beta)$ together with an even bilinear map $\triangleleft: A \otimes M \rightarrow M$, called structure map [11], such that

$$
\begin{aligned}
& A_{a} \triangleleft M_{b} \subseteq M_{a+b} \\
& \beta^{\circ} \triangleleft=\triangleleft^{\circ}(\alpha \otimes \beta), \\
& \triangleleft^{\circ}(\alpha \otimes \triangleleft)=\triangleleft^{\circ}(\mu \otimes \beta) .
\end{aligned}
$$

Definition 5.3: Let $(L,[-,-], \varepsilon, \alpha)$ be a generalized Hom-Lie algebra and $(M, \beta)$ a Hom-module. The G-graded vector space $M$ is said to be a module over $L$, if it is endowed with an even bilinear map [12] $\triangleleft: L \otimes M \rightarrow M$ such that $L_{a} \triangleleft M_{b} \subseteq M_{a+b}, \beta(x \triangleleft m)=\alpha(x) \triangleleft \beta(m)$

and

$$
[x, y] \triangleleft \beta(m)=\alpha(x) \triangleleft(y \triangleleft m)-\varepsilon(x, y) \alpha(y) \triangleleft(x \triangleleft m),
$$

for any $a, b \in G, x, y \in \mathcal{H}(L), m \in \mathcal{H}(M)$.

The following lemma shows that A-modules extend to $L(A)$ modules.

Lemma 5.1: Let $(A, \mu, \varepsilon, \alpha)$ be a generalized Hom-associative algebra and $(M, \beta)$ an $A$-module with structure map $\triangleleft$. Then, $M$ is an $L(A)$-module with the same structure map [11].

Definition 5.4: Let $\left(S,{ }^{\circ}, \mathcal{\varepsilon}, \alpha\right)$ be a generalized left-Homsymmetric algebra. An S-bimodule is a G-graded vector space $M$ endowed with an even linear map $\beta: M \rightarrow M$, two even bilinear maps $S \otimes M \rightarrow M, x \otimes m \mapsto x \prec x$ and $M \otimes S \rightarrow M, m \otimes x \mapsto m \succ x$, such that

$$
S_{a} \prec M_{b} \subseteq M_{a+b}, M_{b} \succ S_{a} \subseteq M_{a+b}, \beta(x \prec m)=\alpha(x) \prec \beta(m), \beta(m \succ x)=\beta(m) \succ \alpha(x)
$$

and

$$
\begin{aligned}
& \alpha(x) \prec(y \prec m)-\left(x^{\circ} y\right) \prec \beta(m)-\varepsilon(x, y)\left(\alpha(y) \prec(x \prec m)-\left(y^{\circ} x\right) \prec \beta(m)\right)=0, \\
& \alpha(x) \prec(m \succ y)-(x \prec m) \succ \alpha(y)-\varepsilon(x, m)\left(\beta(m) \succ\left(x^{\circ} y\right)-(m \succ x) \succ \alpha(y)\right)=0 .
\end{aligned}
$$

Proposition 5.1: Let $\left(S,{ }^{\circ}, \varepsilon, \alpha\right)$ be a generalized left-Homsymmetric algebra and $(M, \prec, \succ, \beta)$ a bimodule over $S$. Then, $M$ is a module over $L(S)$ with the structure map $\triangleleft: S \otimes M \rightarrow M$ defined by

$x \triangleleft m:=x \prec m-\varepsilon(x, m) m \succ x$,

for all $x \in \mathcal{H}(S), m \in \mathcal{H}(M)$.

Proof: For any $x, y \in \mathcal{H}(S), m \in \mathcal{H}(M)$, we have

$[x, y] \triangleleft \beta(m)-\alpha(x) \triangleleft(y \triangleleft m)+\varepsilon(x, y) \alpha(y) \triangleleft(x \triangleleft m)=\left(x^{\circ} y-\varepsilon(x, y) y^{\circ} x\right) \triangleleft \beta(m)$ $-\alpha(x) \triangleleft(y \prec m-\varepsilon(y, m) m \succ y)+\varepsilon(x, y) \alpha(y) \triangleleft(x \prec m-\varepsilon(x, m) m \succ x)$

$=\left(x^{\circ} y-\varepsilon(x, y) y^{\circ} x\right) \prec \beta(m)-\varepsilon(x+y, m) \beta(m) \succ\left(x^{\circ} y-\varepsilon(x, y) y^{\circ} x\right)$

$-\alpha(x) \prec(y \prec m-\varepsilon(y, m) m \succ y)+\varepsilon(x, y+m)(y \prec m-\varepsilon(y, m) m \succ y) \succ \alpha(x)$

$+\varepsilon(x, y) \alpha(y) \prec(x \prec m-\varepsilon(x, m) m \succ x)$

$-\varepsilon(x, y) \varepsilon(y, x+m)(x \prec m-\varepsilon(x, m) m \succ x) \succ \alpha(y)$

$=\underbrace{\left(x^{\circ} y\right) \prec \beta(m)}_{a_{1}}-\underbrace{\varepsilon(x, y)\left(y^{\circ} x\right) \prec \beta(m)}_{a_{2}}-\underbrace{\varepsilon(x+y, m) \beta(m) \succ\left(x^{\circ} y\right)}_{b_{1}}$

$+\underbrace{\varepsilon(x+y, m) \varepsilon(x, y) \beta(m) \succ\left(y^{\circ} x\right)}_{c_{1}}-\underbrace{\alpha(x) \prec(y \prec m)}_{a_{3}}+\underbrace{\varepsilon(y, m) \alpha(x) \prec(m \succ y)}_{b_{2}}$

$+\underbrace{\varepsilon(x, y+m)(y \prec m) \succ \alpha(x)}_{c_{2}}-\underbrace{\varepsilon(x, y+m) \varepsilon(y, m)(m \succ y) \succ \alpha(x)}_{c_{3}}+\underbrace{\varepsilon(x, y) \alpha(y) \prec(x \prec m)}_{a_{4}}$

$-\underbrace{\varepsilon(x, y+m) \alpha(y) \prec(m \succ x)}_{c_{4}}-\underbrace{\varepsilon(y, m)(x \prec m) \succ \alpha(y)}_{b_{3}}+\underbrace{\varepsilon(x+y, m)(m \succ x) \succ \alpha(y)}_{b_{4}}$.

Using (21) observe that $a_{1}+a_{2}+a_{3}+a_{4}=0$, and $b_{1}+b_{2}+b_{3}+b_{4}=0$ and $c_{1}+c_{2}+c_{3}+c_{4}=0$ by (22). The two other relations of the Definition 5.3 , are straight forward proved. This prove that $\mathrm{M}$ is a module over $L(S)$.

Corollary 5.1: Let $\left(S,{ }^{\circ}, \alpha\right)$ be a left-Hom-symmetric algebra and $(M, \prec, \succ, \beta)$ a bimodule over $S$. Then, $M$ is a module over $L(S)$ with the structure map $\triangleleft: S \otimes M \rightarrow M$ defined by

$x \triangleleft m:=x \prec m-m \succ x$,

for all $x \in \mathcal{H}(L), m \in \mathcal{H}(M)$.

Definition 5.5: Let $(D, \dashv, \vdash, \varepsilon, \alpha)$ be a generalized Hom-dendriform algebra. A D-bimodule is a graded vector space $M$ together with an even linear map $\beta: M \rightarrow M$ and four even bilinear maps

$$
\begin{aligned}
D \otimes M \rightarrow M, x \otimes m & \mapsto x \succ m ; & D \otimes M \rightarrow M, x \otimes m & \mapsto x \prec m ; \\
M \otimes D \rightarrow M, m \otimes x & \mapsto m \succ x ; & M \otimes D \rightarrow M, m \otimes x & \mapsto m \prec x
\end{aligned}
$$

such that $D_{a} \prec M_{b} \subseteq M_{a+b}, D_{a} \succ M_{b} \subseteq M_{a+b}, M_{a} \prec D_{b} \subseteq M_{a+b}, M_{a} \succ D_{b} \subseteq M_{a+b}$,

$$
\begin{aligned}
& \beta(x \prec m)=\alpha(x) \prec \beta(m), \beta(x \succ m)=\alpha(x) \succ \beta(m), \\
& \beta(m \prec x)=\beta(m) \prec \alpha(x), \beta(m \succ x)=\beta(m) \succ \alpha(x), \text { and }
\end{aligned}
$$$$
\alpha(x) \succ(y \prec m)=\varepsilon(x, m)(x \vdash y) \prec \beta(m),
$$ 
Citation: Bakayoko I, Diallo OW (2015) Some Generalized Hom-Algebra Structures. J Generalized Lie Theory Appl 9: 226. doi:10.4172/17364337.1000226

Page 6 of 7

$$
\begin{aligned}
& (x \dashv y) \prec \beta(m)=\varepsilon(m, y) \alpha(x) \prec(y \succ m)+\alpha(x) \prec(y \prec m), \\
& \alpha(x) \succ(y \succ m)=\varepsilon(x, y)(x \dashv y) \succ \beta(m)+(x \vdash y) \succ \beta(m), \\
& \alpha(x) \succ(m \prec y)=\varepsilon(x, y)(x \succ m) \prec \alpha(y), \\
& (x \prec m) \prec \alpha(y)=\varepsilon(y, m) \alpha(x) \prec(m \succ y)+\alpha(x) \prec(m \prec y), \\
& \alpha(x) \succ(m \succ y)=\varepsilon(x, m)(x \prec m) \succ \alpha(y)+(x \succ m) \succ \alpha(y), \\
& \beta(m) \succ(x \dashv y)=\varepsilon(m, y)(m \succ x) \prec \alpha(y), \\
& (m \prec x) \prec \alpha(y)=\varepsilon(y, x) \beta(m) \prec(x \vdash y)+\beta(m) \prec(x \dashv y), \\
& \beta(m) \succ(x \vdash y)=\varepsilon(m, x)(m \prec x) \succ \alpha(y)+(m \succ x) \succ \alpha(y) .
\end{aligned}
$$

Proposition 5.2: Let $(M, \prec, \succ, \beta)$ be a bimodule over the generalized Hom-dendriform algebra $(D, \dashv, \vdash, \varepsilon, \alpha)$. Then, $M$ is a bimodule over the generalized left-Hom-symmetric algebra,

associated to $D, S$ ym $(D)$, with the structure map $\triangleleft: D \otimes M \rightarrow M$ defined by

$$
x \triangleleft m:=x \succ m-m \prec x .
$$

Proof: We only need to prove (21) and (22). The other relations being obvious. So, for any $x, y \in \mathcal{H}(D), m \in \mathcal{H}(M)$, we have

$\alpha(x) \triangleleft(y \triangleleft m)-\left(x^{\circ} y\right) \triangleleft \beta(m)-\varepsilon(x, y) \alpha(y) \triangleleft(x \triangleleft m)+\varepsilon(x, y)\left(y^{\circ} x\right) \triangleleft \beta(m)=$

$=\alpha(x) \triangleleft(y \succ m-m \prec y)-(x \vdash y-y \dashv x) \triangleleft \beta(m)$

$-\varepsilon(x, y) \alpha(y) \triangleleft(x \succ m-m \prec x)+\varepsilon(x, y)(y \vdash x-x \dashv y) \triangleleft \beta(m)$

$=\alpha(x) \succ(y \succ m-m \prec y)-(y \succ m-m \prec y) \prec \alpha(x)-(x \vdash y-y \dashv x) \succ \beta(m)$

$+\beta(m) \prec(x \vdash y-y \dashv x)-\varepsilon(x, y) \alpha(y) \succ(x \succ m-m \prec x)+\varepsilon(x, y)(x \succ m-m \prec x) \prec \alpha(y)$

$+\varepsilon(x, y)(y \vdash x-x \dashv y) \succ \beta(m)-\varepsilon(x, y) \beta(m) \prec(y \vdash x-x \dashv y)$

$=\underbrace{\alpha(x) \succ(y \succ m)}_{a_{1}}-\underbrace{\alpha(x) \succ(m \prec y)}_{b_{1}}-\underbrace{(y \succ m) \prec \alpha(x)}_{c_{1}}+\underbrace{(m \prec y) \prec \alpha(x)}_{d_{1}}-\underbrace{(x \vdash y) \succ \beta(m)}_{a_{2}}$

$+\underbrace{(y \dashv x) \succ \beta(m)}_{e_{1}}+\underbrace{\beta(m) \prec(x \vdash y)}_{f_{1}}-\underbrace{\beta(m) \prec(y \dashv x)}_{d_{2}}-\underbrace{\varepsilon(x, y) \alpha(y) \succ(x \succ m)}_{e_{2}}$

$+\underbrace{\varepsilon(x, y) \alpha(y) \succ(m \prec x)}_{c_{2}}+\underbrace{\varepsilon(x, y)(x \succ m) \prec \alpha(y)}_{b_{2}}-\underbrace{\varepsilon(x, y)(m \prec x) \prec \alpha(y)}_{f_{2}}$

$+\underbrace{\varepsilon(x, y)(y \vdash x) \succ \beta(m)}_{e_{3}}-\underbrace{\varepsilon(x, y)(x \dashv y) \succ \beta(m)}_{a_{3}}-\underbrace{\varepsilon(x, y) \beta(m) \prec(y \vdash x)}_{d_{3}}$

$+\underbrace{\varepsilon(x, y) \beta(m) \prec(x \dashv y)}_{f_{3}}$.

Observe that $a_{1}+a_{2}+a_{3}$ cancels by (30), $b_{1}+b_{2}$ cancels by (31), $c_{1}$ $+c_{2}$ cancels by (31), $d_{1}+d_{2}+d_{3}$ cancels by (35), $e_{1}+e_{2}+e_{3}$ cancels by (30) and $f_{1}+f_{2}+f_{3}$ cancels by (35).

Next,

$\alpha(x) \triangleleft(m \triangleright y)-(x \triangleleft m) \triangleright \alpha(y)-\varepsilon(x, m) \beta(m) \triangleright\left(x^{\circ} y\right)+\varepsilon(x, m)(m \triangleright x) \triangleright \alpha(y)=$

$=\alpha(x) \triangleleft(m \succ y-y \prec m)-(x \succ m-m \prec x) \triangleright \alpha(y)$

$-\varepsilon(x, m) \beta(m) \triangleright(x \vdash y-y \dashv x)+\varepsilon(x, m)(m \succ x-x \prec m) \triangleright \alpha(y)$

$=\alpha(x) \succ(m \succ y-y \prec m)-(m \succ y-y \prec m) \prec \alpha(x)-(x \succ m-m \prec x) \succ \alpha(y)$

$+\alpha(y) \prec(x \succ m-m \prec x)-\varepsilon(x, m) \beta(m) \succ(x \vdash y-y \dashv x)+\varepsilon(x, m)(x \vdash y-y \dashv x) \prec \beta(m)$,

$$
\begin{aligned}
& +\varepsilon(x, m)(m \succ x-x \prec m) \succ \alpha(y)-\varepsilon(x, m) \alpha(y) \prec(m \succ x-x \prec m) \\
& =\underbrace{\alpha(x) \succ(m \succ y)}_{a_{1}}-\underbrace{\alpha(x) \succ(y \prec m)}_{b_{1}}-\underbrace{(m \succ y) \prec \alpha(x)}_{c_{1}}+\underbrace{(y \prec m) \prec \alpha(x)}_{d_{1}}-\underbrace{(x \succ m) \succ \alpha(y)}_{a_{2}} \\
& +\underbrace{(m \prec x) \succ \alpha(y)}_{e_{1}}+\underbrace{\alpha(y) \prec(x \succ m)}_{f_{1}}-\underbrace{\alpha(y) \prec(m \prec x)}_{d_{2}}-\underbrace{\varepsilon(x, m) \beta(m) \succ(x \vdash y)}_{e_{2}} \\
& +\underbrace{\varepsilon(x, m) \beta(m) \succ(y \dashv x)}_{c_{2}}+\underbrace{\varepsilon(x, m)(x \vdash y) \prec \beta(m)}_{b_{2}}-\underbrace{\varepsilon(x, m)(y \dashv x) \prec \beta(m)}_{f_{2}} \\
& +\underbrace{\varepsilon(x, m)(m \succ x) \succ \alpha(y)}_{e_{3}}-\underbrace{\varepsilon(x, m)(x \prec m) \succ \alpha(y)}_{a_{3}}-\underbrace{\varepsilon(x, m) \alpha(y) \prec(m \succ x)}_{d_{3}} \\
& +\underbrace{\varepsilon(x, m) \alpha(y) \prec(x \prec m)}_{f_{3}} .
\end{aligned}
$$

Observe that $a_{1}+a_{2}+a_{3}=0$ by (33), $b_{1}+b_{2}=0$ by (28), $c_{1}+c_{2}=0$ by (34), $d_{1}+d_{2}+d_{3}=0$ by (32), $e_{1}+e_{2}+e_{3}=0$ by (36) and $f_{1}+f_{2}+f_{3}=0$ by (29).

Corollary 5.2: Let $(M, \prec, \succ, \beta)$ be a bimodule over the Homdendriform $(D, \dashv, \vdash, \alpha)$. Then, $M$ is a bimodule over the left-Homsymmetric algebra Sym(D) associated to $D$.

Definition 5.6: Let $(A, \cdot, \alpha)$ be a Hom-associative algebra and let $(M$, $\beta)$ be a Hom-module. A bimodule structure on $M$ consists of:

i) a left $A$-action $\prec: A \otimes M \rightarrow M(a \otimes m \mapsto a \prec m)$, and

ii) a right $A$-action $\succ: M \otimes A \rightarrow M(m \otimes a \mapsto m \succ a)$ such that the following conditions hold for $x, y \in A$ and $m \in M$ :

$$
\begin{aligned}
& \beta(x \prec m)=\alpha(x) \prec \beta(m), \\
& \beta(m \succ x)=\beta(m) \succ \alpha(x), \\
& \alpha(x) \prec(y \prec m)=(x \cdot y) \prec \beta(m), \\
& (m \succ x) \succ \alpha(y)=\beta(m) \succ(x \cdot y), \\
& \alpha(x) \prec(m \succ y)=(x \prec m) \succ \alpha(y) .
\end{aligned}
$$

Proposition 5.3: Let $(M, \prec, \succ, \beta)$ be a bimodule over the Homdendriform $(D, \dashv, \vdash, \alpha)$. Then, $M$ is a bimodule over the Hom-associative algebra associated to $D, A s s(D)$, with the structure map

$x \triangleleft m=x \succ m+x \prec m, \quad m \triangleright x=m \succ x+m \prec x$

Proof: For any $x, y \in \mathcal{H}(D), m \in \mathcal{H}(M)$, we have

$\beta(x \triangleleft m)=\beta(x \prec m+x \succ m)=\alpha(x) \prec \beta(m)+\alpha(x) \succ \beta(m)=\alpha(x) \triangleleft \beta(m)$,

$\beta(m \triangleright x)=\beta(m \prec x+m \succ x)=\beta(m) \prec \alpha(x)+\beta(m) \succ \alpha(x)=\beta(m) \triangleright \alpha(x)$.

Then,

$\alpha(x) \triangleleft(y \triangleleft m)-(x \star y) \triangleleft \beta(m)=\alpha(x) \triangleleft(y \succ m+y \prec m)-(x \vdash y+x \dashv y) \triangleleft \beta(m)=$

$$
\begin{aligned}
& =\alpha(x) \succ(y \succ m+y \prec m)+\alpha(x) \prec(y \succ m+y \prec m) \\
& -(x \vdash y+x \dashv y) \succ \beta(m)-(x \vdash y+x \dashv y) \prec \beta(m) \\
& =\alpha(x) \succ(y \succ m)+\alpha(x) \succ(y \prec m)+\alpha(x) \prec(y \succ m)+\alpha(x) \prec(y \prec m) \\
& -(x \vdash y) \succ \beta(m)-(x \dashv y) \succ \beta(m)-(x \vdash y) \prec \beta(m)-(x \dashv y) \prec \beta(m)
\end{aligned}
$$

The left hand side vanishes by axioms (28)-(30), with trivial grading. Next,

$(m \triangleright x) \triangleright \alpha(y)-\beta(m) \triangleright(x \star y)=(m \succ x+m \prec x) \triangleright \alpha(y)-\beta(m) \triangleright(x \vdash y+x \dashv y)$ 


$$
\begin{aligned}
& =(m \succ x+m \prec x) \prec \alpha(y)+(m \succ x+m \prec x) \succ \alpha(y) \\
& -\beta(m) \prec(x \vdash y+x \dashv y)-\beta(m) \succ(x \vdash y+x \dashv y) \\
& =(m \succ x) \prec \alpha(y)+(m \prec x) \prec \alpha(y)+(m \succ x) \succ \alpha(y)+(m \prec x) \succ \alpha(y) \\
& -\beta(m) \prec(x \vdash y)-\beta(m) \prec(x \dashv y)-\beta(m) \succ(x \vdash y)-\beta(m) \succ(x \dashv y)
\end{aligned}
$$

The left hand side vanishes by axioms (34)-(36), with trivial grading. Finally,

$$
\begin{aligned}
& \alpha(x) \triangleleft(m \triangleright y)-(x \triangleleft m) \triangleright \alpha(y)= \\
& =\alpha(x) \prec(m \succ y+m \prec y)+\alpha(x) \succ(m \succ y+m \prec y) \\
& -(x \succ m+x \prec m) \prec \alpha(y)-(x \succ m+x \prec m) \succ \alpha(y) \\
& =\alpha(x) \prec(m \prec y)+\alpha(x) \prec(m \succ y)+\alpha(x) \succ(m \prec y)+\alpha(x) \succ(m \succ y) \\
& -(x \prec m) \prec \alpha(y)-(x \succ m) \prec \alpha(y)-(x \prec m) \succ \alpha(y)-(x \succ m) \succ \alpha(y)
\end{aligned}
$$

The left hand side vanishes by axioms (31)-(33), with trivial grading.

Now, let us denote by HDbiM: the category of bimodules over Hom-dendriform algebra, LHSbiM: the category of bimodules over left-Hom-symmetric algebra, HABiM: the category of bimodules over Hom-associative algebra and HLM: the category of modules over Hom-Lie algebra. Then, we have:

Theorem 5.1: The following diagram of categories and functors is commutative

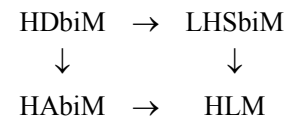

Proof: The top horizontal arrow and the bottom horizontal arrow follow from Corollary 5.2 and Lemma 5.1 respectively. The left vertical arrow is established in Proposition 5.3 and right vertical arrow is the functor constructed in Corollary 5.1.

\section{References}

1. Aizawa N, Sato H (1991) Q-deformation of the Virasoro algebra with centra extension. Physics Letters 256: 185-190.
2. Makhlouf A Silvestrov S (2010) Notes on formal deformations of Homassociative algebras and Hom-Lie algebras. Forum Math 22: 715-739.

3. Ammar F, Mabrouk S, Makhlouf A (2011) Quadratic n-ary Hom-Nambu algebras.

4. Berrick AJ, Keating ME (2000) Rings and modules. An introduction with K-theory in view Cambridge University Press, USA.

5. Yau D (2012) Hom-Maltsev, Hom-alternative and Hom-Jordan algebras. Int Elect Journ of Alg 11: 177-217.

6. Bakayoko I (2014) L-modules, L-comodules and Hom-Lie quasi-bialgebras African Diaspora Journal of Mathematics 17: 49-64.

7. Bakayoko I (2014) Laplacian of Hom-Lie quasi-bialgebras. Inter Journ of Alg 8: 713-727.

8. Elhamdadi M, Makhlouf A (2010) Deformations of Hom-Alternative and HomMalcev algebras.

9. Abdaoui K, Ammar F, Makhlouf A (2013) Constructions and cohomology of generalized Hom-Lie Algebras.

10. Bakayoko I, Oh SQ. Color Hom-Lie bialgebras and color Hom-Poisson bialgebras.

11. Bakayoko I (2014) Modules over color Hom-Poisson algebras. J Gen Lie Theory Appl 8: 1-6.

12. Yuan $L$ (2012) Hom-Lie color algebras. Communication in algebra 40: 575-592.

13. Ammar F, Makhlouf A (2010) Hom-Lie superalgebras and Hom-Lie admissible super- algebras. Journal of Algebra 324: 1513-1528.

14. Ammar F, Ayadi I, Mabrouk S, Makhlouf A (2012) Quadratique color Hom-Lie algebras.

15. Scheunert M, Zhang RB (1998) Cohomology of Lie superalgebras and their generalizations. Journal of Mathematical Physics 39: 5024-5061.

16. Ammar F, Saadaoui N, Makhlouf A (2011) Cohomology of Hom-Lie superalgebras and q-deformed Witt superalgebra. To appear in Czechoslovak Mathematical Journal 63: 721-761.

17. Zhang RX (2013) Cohomologies and deformations of generalized leftsymmetric algebras.

18. Aguiar M (2002) Infinitesimal bialgebras, pre-Lie and dendriform algebras.

19. Makhlouf A (2011) Hom-dendriform algebras and Rota-baxter algebras

20. Bakayoko I, Bangoura M. Left-Hom-symmetric algebras and Hom-Poisson dialgebras.
Citation: Bakayoko I, Diallo OW (2015) Some Generalized Hom-Algebra Structures. J Generalized Lie Theory Appl 9: 226. doi:10.4172/1736 4337.1000226
Submit your next manuscript and get advantages of OMICS Group submissions

Unique features:

- User friendly/feasible website-translation of your paper to 50 world's leading languages

- Audio Version of published paper

Digital articles to share and explore

Special features:

400 Open Access Journals

30,000 editorial team

21 days rapid review process

Quality and quick editorial, review and publication processing

Indexing at PubMed (partial), Scopus, EBSCO, Index Copernicus and Google Scholar etc

Sharing Option: Social Networking Enabled

- Authors, Reviewers and Editors rewarded with online Scientific Credits

Better discount for your subsequent articles

Submit your manuscript at: http://www.omicsonline.org/submission 